\title{
The effect of movement variability on putting proficiency during the golf putting stroke
}

Ashley K. Richardson

Andrew C. S. Mitchell

Gerwyn Hughes

Richardson, A.K., Mitchell, A.C.S. and Huhes. The effect of movement variability on putting proficiency during the putting stroke, International Journal of Sports Science and Coaching. (C) the authors 2018. Reprinted by permission of SAGE Publications.

DOI: https://doi.org/10.1177/1747954118768234 
1 The effect of movement variability on putting proficiency during the golf putting

2 stroke

3 Ashley K. Richardson ${ }^{1}$, Andrew C. S. Mitchell ${ }^{2}$, \& Gerwyn Hughes ${ }^{3}$

$4 \quad{ }^{1}$ Division of Sport and Exercise Sciences, School of Social and Health Sciences, Abertay

5 University, UK. ${ }^{2}$ School of Sport Science and Physical Activity, Faculty of Education and

6 Sport, University of Bedfordshire, UK. ${ }^{3}$ Department of Kinesiology, College of Arts and

7 Sciences, University of San Francisco, USA.

8 KEYWORDS: golf putting, variability, performance, kinematics, dynamical systems, golf

9 coaching

10

11

12

13

14

15

16

17

18

19 
21 Movement variability has been considered important to execute an effective golf swing 22 yet is comparatively unexplored regarding the golf putt. Movement variability could 23 potentially be important considering the small margins of error between a successful and 24 a missed putt. The aim of this study was to assess whether variability of body segment 25 rotations influence putting performance (ball kinematic measures). Eight golfers 26 (handicap range 0 - 10) performed a 3.2 metre level putt wearing retro-reflective markers 27 which were tracked using a three-dimensional motion analysis system sampling at 120 


\section{Introduction}

43 The putting stroke accounted for $41 \%$ of all strokes during tournaments on the

44 Professional Golf Association (PGA) Tour in 2014. 1,2 Additionally putting is a key

45 determinate of earnings on the PGA Tour. ${ }^{3,4}$ Recently movement variability has been

46 identified as an important biomechanical principle to research. ${ }^{5-7}$ Currently to date

47 research of movement variability in the golf putt is scarce with more research needed in

48 the area to establish its effect on performance. ${ }^{8}$ Movement variability has been stated as

49 important for successful performance and technique during the golf swing. ${ }^{5,9}$ Considering

50 similar performance goals for golf putting movement variability may also be important for

51 this aspect of golf.

52 As outlined in dynamical systems theory, movement patterns arise, mature and develop

53 from synergistic organisation of the neuromuscular system adapting to environmental

54 factors exposed to, morphological factors and task constraints. ${ }^{10}$ Different movement

55 patterns will develop between individuals with a unique set of different constraints,

56 allowing for different techniques to achieve the same performance outcome. ${ }^{11,12}$ With the

57 golf swing being a complex and high velocity technique the existence of an invariant

58 movement pattern is unlikely. ${ }^{5}$ Inter and intra-individual differences may also be apparent

59 for the golf putt, due to the smaller margins of error between a successful or missed shot.

6013,14 The consensus amongst the literature with the full golf swing in regard to movement

61 variability is to reduce variability at key swing events for successful performance. ${ }^{9,15,16}$

62 The authors however consider using a time-continuous data set for the calculation of 63 variability preferable to observing variability at specific points. ${ }^{7}$ This is because the golf 64 swing or putting stroke is a continuous skill and doesn't occur only at discrete points, 
therefore it is more applicable to observe variability across the full movement. When variability across the golf swing from the start of the movement to impact was considered in the full golf swing, no relationship with an outcome measure (initial velocity of the golf ball) was identified. ${ }^{5} \mathrm{~A}$ limitation of the aforementioned study was ball direction or accuracy was not considered a performance measure. Movement variability will likely affect the swing trajectories and club head angle at impact (affecting shot direction) as well as the speed of movement (affecting the ball flight velocity). Club head angle at impact has previously shown variability for the golf swing and golf putt. ${ }^{13,14,17}$

During putting it has been established that factors accounting for direction consistency/variability - putter face angle $(80-83 \%)$, the trajectory of the putter path $(17 \%)$ and horizontal impact point on the putter face (3\%). ${ }^{14,18}$ In principle if these task criterion factors remain consistent with a low variability the initial launch angle of the golf ball will remain consistent resulting in more putts that are successful. When considering technique it should matter little as to whether a consistent technique with low variability, or coordinated variability of body movement is utilised to achieve this. Therefore, emphasis always being placed on a low variability movement may be incorrect when considered from a dynamical systems approach and different strategies including variable body movement patterns may be integral to successful putting performance. ${ }^{5,19}$

Movement variability for some may be a key determining factor to the reduction in variance of the task criterion putter face angle at impact and therefore performance. Coaching and golf putting instruction manuals traditionally has focused on encouraging techniques aiming to achieve low variability, where a linear stroke is desired. ${ }^{18}$ Scientific literature has however outlined this is biomechanically complicated and difficult with 
88 reliance on compensatory muscle activity keeping the putter face square whilst the body

89 rotates. ${ }^{14}$ This therefore may not be the best technique for golfers to adopt or coaches to 90 teach.

91 The aim of this study was to assess whether the variability of body segment rotations and

92 putter rotations influence the variance of performance measures (ball roll kinematics:

93 velocity, side spin, initial ball roll, forward rotation, vertical launch angle and horizontal

94 launch angle). It was hypothesised that significant relationships would exist between the 95 variability of body segments and performance measures.

\section{Methods}

\section{Participants}

98 A total of 8 right-handed golfers participated in the study (age $34 \pm 11$ years; handicap

$996.0 \pm 3.4$ (handicap range $0-10$ ); height $1.80 \pm 0.06$ metres; mass $83.4 \pm 12.2 \mathrm{~kg}$ ). All 100 golfers were free of musculoskeletal injury for a minimum period of 3 months and played 101 a minimum of once a week. During testing participants wore their own personal golfing 102 attire and suitable dark, tight fitting non-reflective shorts and short sleeved top. All 103 participants provided written informed consent and the study was approved by the 104 institutional ethics committee of University of Hertfordshire.

106 Experimental set-up

107 Testing was completed on a Huxley Golf (Huxley Golf., Hampshire, UK) artificial putting 108 green (3.66 x 4.27 metres) registering 11 on the stimpmeter (The United States Golf 
Association., Far Hills, NJ, USA). A level, straight 3.2 metre putt was setup thus minimising the effect of green reading and aim with a regulation $108 \mathrm{~mm}$ hole. ${ }^{14,20}$ Participants used their own personal putter for the protocol. The rationale for this was the participant would be using a putter they were already habituated to. This ensured the body movement kinematics were a true reflection of their technique, whereas a standardised putter not fitted to each of the participants could negatively influence this. The golf ball for the protocol were Srixon Z-STAR (Srixon Sports Europe LTD., Hampshire, UK) and each trial completed used the same ball. Body movement kinematics were recorded using a ten camera motion analysis system (Motion Analysis Corporation., Santa Rosa, CA, USA) sampling at $120 \mathrm{~Hz}$.

Retro-reflective markers were attached to participants in accordance with a modified whole body Helen Hayes marker set (total 31 markers; $14 \mathrm{~mm}$ ) at the following anatomical locations: top of head, front of head, rear head, acromion process (left and right), lateral epicondyle of humerus (left and right), styloid process of the radius (left and right), on the forearm intersecting the humeral epicondyle and styloid process of the radius (left and right), anterior superior iliac spine (left and right), the sacrum, the thigh (intersecting the plane between the hip and knee markers (left and right)), lateral aspect of the joint centre of the knee (left and right), the shank (intersecting the plane between the knee and ankle markers (left and right)), the lateral malleolus (left and right), the posterior aspect of the calcaneus (left and right) and the third metatarsal (left and right). Markers were placed directly on the skin using double sided tape, except the acromion process (pair of), anterior superior iliac spine (pair of), sacrum, calcaneus (pair of) and third metatarsal (pair of) which were placed on skin tight 'under-armour' clothing or shoes ensuring minimal 
132 movement of markers relative to underlying body landmarks. Additionally, a marker was 133 placed on the left scapula for asymmetry and medial aspects of the knee (left and right) 134 and medial malleolus (left and right) so the joint centres of the knee and ankle could be 135 calculated.

136 Two retro-reflective markers were placed on the superior aspect of the putter face to 137 calculate putter face angle at impact and throughout the putting stroke. A retro-reflective 138 marker was also placed on the putting line. The capture volume was calibrated according 139 to manufacturer's guidelines, resulting in an average residual for all cameras of $<0.2 \mathrm{~mm}$. 140 The motion analysis system was calibrated where the positive movement along the X141 axis was defined as movement towards the target (golf hole); positive movement along 142 the $\mathrm{Y}$-axis was defined as movement anteriorly perpendicular to the target; and the $\mathrm{Z}$-axis 143 perpendicular to the $\mathrm{X}, \mathrm{Y}$ plane.

144 To record the ball roll kinematics, a Quintic (Quintic Consultancy Ltd., Coventry, UK) high 145 speed camera (Ul-5220RE) sampling at $220 \mathrm{~Hz}$ was positioned perpendicular to the 146 putting line. The Quintic v2.4 launch monitor software was used to analyse the recorded 147 ball roll. Kinematic variables analysed were initial velocity $\left(\mathrm{m} \cdot \mathrm{s}^{-1}\right.$, calculated across the 148 first 6 recorded frames), side spin (the amount of side spin (rpm) placed on the ball during 149 impact), vertical (whether the ball was launched in the air) and horizontal (the degree to 150 which the ball deviates from the original putting line) launch angle $(\stackrel{0}{*})$, initial ball roll 151 (whether the ball has positive rotation (topspin) or negative rotation (backspin) at the point 152 of impact (rpm)) and forward roll (the distance at which the ball starts positive rotation $153(\mathrm{~cm})$ ). For a trial to be considered valid, the initial ball velocity had to be between $2.10-$ $1542.28 \mathrm{~m} \cdot \mathrm{s}^{-1}$. This was to eliminate participants' preference of either putting to hole the ball 
155 successfully at very low or high velocities which could alter movement variability 156 observed. Putts that did not meet the initial ball velocity requirements were eliminated 157 from analysis. Despite this only one putt was eliminated from analysis.

\section{Procedure}

160 Participants were allowed up to ten minutes to habituate themselves to the golf putt, to 161 ensure that the markers did not inhibit or alter their technique. Within the ten minute

162 habituation period, the investigator instructed the participant as to the velocity required 163 for a putt to be categorised successful. Once the participant was comfortable and ready 164 to proceed, they lined up the golf putt and approached the putt. The 3D motion analysis 165 system recorded the trial and the outcome of the putt was recorded (successful or 166 missed). This process was completed until 10 successful putts had been completed with 167 time between each trial for the participant to reline up the putt. All putts (successful and 168 unsuccessful) were included for analysis.

\section{Data Processing}

171 Three-dimensional coordinate data were processed using Cortex (Motion Analysis

172 Corportation; Santa Rosa, CA, USA) software with an Euler sequence of $\mathrm{X}, \mathrm{Y}, \mathrm{Z}$. The 3D

173 coordinate data were filtered using a fourth-order low pass Butterworth filter, consistent 174 with previously published literature. ${ }^{16,21,22}$ Cut off frequency was determined using 175 residual analysis with an $r^{2}$ threshold of $0.85 .{ }^{23}$ Cut off frequencies used for the markers 176 ranged from $6-8 \mathrm{~Hz}$. Due to intra and inter subject differences in the duration of trials, 
$1773 \mathrm{D}$ segmental coordinates and putter rotations were time-normalised to 101 data points 178 using a cubic spline algorithm. The section of the golf putt that was normalised was from

179 the first movement during the putt backswing until the point of impact with the golf ball, 180 the follow-through was not used for analysis. This allowed for accurate means and 181 variation to be calculated. Following this, kinematic data were processed into segments.

182 Performance variability was calculated for all body segments as outlined previously within 183 golf literature. ${ }^{5}$ Rotations were normalised to the position at address one frame before 184 the trial started. Following this normalisation process, the standard deviation was 185 calculated for the 101 data points for all the trials of each participant's $X, Y$ and $Z$ 186 coordinates. These were then combined via multiplication to have a single number 187 represent the 3D rotational variability. ${ }^{5,24}$ The equation below was used to calculate a 188 scalene ellipsoid for each participant representing the 3D variability of the rotations for 189 the 101 data points. ${ }^{5}$ This was then averaged to give a mean variability volume $190\left(\right.$ degrees $\left.^{3}\right)$ :

$$
V V=\frac{\sum_{n=1}^{101} \frac{4}{3} \pi\left(s d_{x i} \cdot s d_{y i} \cdot s d_{z i}\right)}{101}
$$

192 where $\mathrm{VV}$ is the mean variability for each segments rotation, [sdxi, $s d_{y i}, s d_{z i}$ are the 193 standard deviations for all planes of movement at point $i$. When interpreting the mean 194 variability score (VV), it was important to consider the range of rotation for each of the 195 segments. Therefore, the mean variability score was standardised to the 3D rotations. 196 The calculation used to calculate the average 3D distance over the trials (degrees) were:

$$
\mathrm{PD}=\left(\sum_{i=1}^{101} \sqrt{\left(x_{i+1}-x_{i}\right)^{2}+\left(y_{i+1}-y_{i}\right)^{2}+\left(z_{i+1}-z_{i}\right)^{2}}\right)
$$


198 where PD is the performance distance of each segment, $\left[\mathrm{x}_{i}, \mathrm{y}_{\mathrm{i}}, \mathrm{z}_{\mathrm{i}}\right]$ and $\left[\mathrm{x}_{\mathrm{i}+1}, \mathrm{y}_{i+1}, \mathrm{z}_{i+1}\right]$ are 199 the positions at a point $i$ during the trial and point $i+1$. This was adapted from previous 200 literature that has calculated movement variability. ${ }^{5}$ Performance variability was defined 201 as the mean variability volume divided by the performance distance:

$$
P V=\frac{V V}{P D}
$$

203

204

205

206

207

where PV is termed the performance variability. ${ }^{5}$ This provided a volume per distance measure (degrees $3 /$ degrees). The only segment that was analysed in a different fashion was the putter segment where only $Z$ rotations were recorded, therefore the standard deviations were totalled and normalised by the $\mathbf{Z}$ rotations displacement.

\section{Data Analysis}

Segmental rotations $\left({ }^{\circ}\right)(\mathrm{X}, \mathrm{Y}$ and $\mathrm{Z})$ were formulated for the pelvis, torso, left and right upper arm and left and right lower arm. These segments were selected as they have previously been analysed and are thought to contribute to the impulse being imparted on the ball during the putt. ${ }^{18,25,26}$ Ball kinematic variables measured were: velocity (velocity $(\mathrm{m} / \mathrm{s})$ of the ball during the first 6 frames captured), side spin (cut or hook (the amount of side spin (rpm) placed on the ball during impact)), initial ball roll (whether the golf ball had positive rotation (topspin) or negative rotation (backspin) at the point of impact), forward roll (the distance at which the ball is rolling in a positive direction), vertical launch angle (the launch angle at the point of impact on the vertical axis) and the horizontal launch angle (the launch angle at the point of impact on the horizontal axis). Associations between performance variability for body segment rotation and outcome variability (ball 
220 kinematic variables) were calculated and outcome variability was tested as a coefficient

221 of variation (\%). ${ }^{5}$

223 Data were exported to the statistical software package SPSS v23 (SPSS Inc, Chicago, 224 USA) for analysis. The data were analysed for normality using a Shapiro-Wilk test of 225 normality and assessment of kurtosis and skewness values. The data were found to be 226 non-parametric and therefore a Spearman's rank correlation coefficient test was carried 227 out. The boundaries set for the coefficient statistics were; $r=0.8-1.0$, very strong, $r=$ $2280.6-0.8$, strong, $r=0.4-0.6$, moderate, $r=0.2-0.4$, weak, $r=0.0-0.2$, no relationship. 229 Level of significance was set at $\alpha<0.05$.

\section{Results}

231 Individual performance variability for the segment rotations are presented in Figure 1. A 232 range of variability was observed, the largest being 0.74 degrees $3 /$ degrees for participant 233 one. Participant eight demonstrated virtually no segment variability suggesting a very 234 consistent movement pattern.

Putter variation, variation of putter face angle at address and putting proficiency are 239 presented in Table 1. Participant eight displayed the best putting proficiency (83\%) this was coupled with one of the lower performance variability scores for the putter $(0.17$ 
241 degrees $3 /$ degrees). A range of correlations were observed between segment variability,

242 putter face angle at address and putting proficiency (putter and putting proficiency; no

243 association, left forearm and putting proficiency; moderate association, right upper arm

244 and putting proficiency; strong association). However, all correlations were identified all 245 to be non-significant (Table 2).

247 TABLE 1 \& 2 ABOUT HERE

249 Mean ball roll kinematic results are presented in Table 3 and correlation coefficients 250 between performance measurement variability (ball roll kinematics) and segment rotation

251 variability in Table 4. One significant correlation was identified between segment 252 variability of the left forearm and variability of the horizontal launch angle of the golf ball $253(r=.92$ (very strong association), $p<.01)$. Additionally, near significant positive 254 relationship was identified for the variability of the pelvis and horizontal launch angle $(r=$ 255.65 (strong association), $p=.08$ ).

256

257 TABLE 3 \& 4 ABOUT HERE

258

259 Discussion

260 This is one of the first studies to have considered body segment variability during the golf 261 putting stroke. It was hypothesised that significant relationships would exist between the 
262 variability of body segments and variability of performance measures. This hypothesis

263 can predominantly be rejected with no significant relationships identified between

264 segment variability and putting proficiency and only one significant correlation identified

265 between the variability of the horizontal launch angle and variability of the left forearm

266 segment.

267 Within golf to date variability has only been considered for the full golf swing. $5,9,15,16$

268 Despite this, the desired outcome for the putt is very similar to the full swing; a shot that

269 is accurate with the correct amount of power applied. Therefore, to obtain this sought

270 after outcome, theoretically, a movement system must be a balance of stable (persistent)

271 and flexible motor outputs, allowing the golfer to adapt to the requirements of the shot.

27211,15 It was also found no relation between performance variability and ball velocity

273 variability, concluding that individual players use their own strategies to control

274 performance variability so it did not affect outcome variability. ${ }^{5}$ The results of the current

275 study suggest this is also evident for the golf putt. With no significant correlations identified

276 between variability of segments and putting proficiency suggest some golfers within the

277 current study utilised or controlled performance variability to minimise output variability.

278 Therefore, less variability isn't necessarily desirable for all golfers, with some golfers able

279 to still putt successfully despite demonstrating more variability than others. For example

280 participant one showed the second largest variability of the left forearm and largest

281 variability of the pelvis (Figure 1) and had a 73\% success rate. Whereas, in comparison

282 participant seven demonstrated less segment variability and had a $67 \%$ rate, less than

283 that of participant one and participant three who demonstrated low performance variability

284 and was the worst performing golfer (52\%). The most successful golfer (83\%) participant 
285 eight demonstrated virtually no movement variability, emphasising the individual 286 approaches observed in the current study.

287 It has been reported that a reduction in the variability of the hand trajectory from mid288 downswing to impact improved performance for the full golf swing. ${ }^{16}$ This however 289 contrasting evidence exists with increased variability observed during the downswing 290 phase. ${ }^{5}$ Results from the current study (analysed to impact) shows that segment 291 variability of the left forearm increased the variability of the horizontal launch angle with a 292 very strong relationship observed (Table 4). This suggests that players that demonstrate 293 less variability will see better performances, as in the ball starts travel in the intended 294 direction. However, this did not translate to a positive relationship in variability of the left 295 forearm and putting proficiency with a non-significant moderate association observed. A 296 potential explanation for this may be the additional variability observed at address $(0.48$ $297-1.77$ degrees, Table 1). Previously, the putter face angle has been deemed to be 298 essential regarding the initial direction of the golf putt. ${ }^{13,14,18}$ Across the studies a range 299 of $80-95 \%$ of the starting direction (horizontal launch angle) of a putt was accredited to 300 putter face angle. ${ }^{13,14,18}$ It may be the case variability of the putter face angle may 301 accommodate some variability of the angle at address. Demonstrating that performance 302 variability may not be detrimental to performance, whereby a different combination of 303 rotations result in a square putter face at impact is equally as desirable as minimal 304 variability. Another factor that could have influenced results were the range of initial ball 305 velocity range the participants were instructed to follow. However, no participants 306 mentioned this as an issue or factor they considered when completing the protocol. 
307 Previously it has been observed greater movement variability of the pelvis and trunk in 308 less proficient golfers $(<79 \%$ success rate) in comparison to more proficient golfers ( $>$ $30979 \%$ success rate). ${ }^{25}$ The current study's results are in contrast to this. Golfers in the 310 current study demonstrated a consistent variability of the pelvis $(0.01-0.74$ 311 degrees $3 /$ degrees $)$ and trunk $\left(0.00-0.09\right.$ degrees $^{3} /$ degrees $)$. This includes participant 312 one who demonstrated increased variability of the pelvis in comparison to the other 313 participants and was not the worst performing golfer (Figure 1). Additionally, no significant 314 correlations were observed for performance variability of the pelvis $(r=-.44$; moderate 315 association) and trunk ( $r=-.38$; weak association) with putting success rate (Table 2).

316 Differences between the two studies may be due to the analysis techniques, whereby 317 individual putting events during the stroke were assessed whereas the current study 318 totalled variation for all three planes and normalised the data by the rotational 319 displacement of each segment. It also may be due to the large intra and inter-subject 320 variability observed in both this study and the previous article that differences actually 321 existed between each study. ${ }^{25}$

322 It is proposed by the authors of the current study that different styles of putting may be 323 employed by golfers. Whereby some utilise more stable motor outputs (participant eight) 324 whereas others utilise more flexible motor outputs (participant one). More research into 325 movement variability and putting is needed to confirm this however. This study 326 additionally provides support for previous biomechanical literature that it is beneficial for 327 individual based analysis within biomechanical golf analysis. ${ }^{5,27}$ Future research needs 328 to test a larger number of highly skilled participants to determine whether different styles 329 of putting exist when considering movement variability. Based on the results of the current 
330 study the practical implications of the study are golf coaches should aim to ascertain 331 whether the golfer utilises movement variability or has a consistent movement pattern and

332 refine their current technique. It may not be beneficial to teach a new consistent putting 333 style.

\section{Conclusion}

335 This is the one of the first studies to have considered movement variability effect on 336 performance measures in the golf putt. It was established that there was no relationship 337 between putting proficiency and performance (segment) variability. One significant 338 relationship was observed between left forearm variability and the horizontal launch angle 339 of the ball but this was not a detriment to performance, this may have been because of 340 the variability of the putter face angle at initial setup. Considering the statistically non341 significant results it is postulated that different styles of golf putting may exist; one 342 whereby more stable motor outputs are utilised and secondly where more flexible motor 343 outputs are adopted. We emphasise the need to include individual based analysis in 344 future biomechanical golf studies. Golf coaches should aim to determine whether 345 movement variability is being utilised where output variability is not affected resulting in 346 equally effective performance as a golfer who demonstrates a highly consistent 347 movement pattern.

349 Acknowledgements

350 The authors would like to thank the University of Hertfordshire for providing facilities and 351 ethical approval to complete this research. 


\section{References}

353 1. PGA Tour. 2014 PGA TOUR scoring average

354

(actual)http://www.pgatour.com/stats/stat.120.2014.html (2015, accessed 28 June 2015).

2. PGA Tour. 2014 PGA TOUR putts per

roundhttp://www.pgatour.com/stats/stat.119.html (2015, accessed 28 June 2015).

3. Alexander DL, Kern W. Drive for show and putt for dough? An analysis of the earnings of PGA tour golfers. J Sports Econom 2005; 6: 46-60.

4. Dorsel TN, Rotunda RJ. Low scores top 10 finishes, and big money: An analysis of Professional Golf Association Tour statistics and how these relate to overall performance. Percept Mot Skills 2001; 92: 575-585.

5. Tucker CB, Anderson R, Kenny IC. Is outcome related to movement variability in golf? Sport Biomech 2013; 12: 343-354.

6. Farrally MR, Cochran AJ, Crews DJ, et al. Golf science research at the beginning of the twenty-first century. J Sports Sci 2003; 21: 753-765.

7. Glazier P. Movement Variability in the Golf Swing. Res Q Exerc Sport 2011; 82:

8. Gryc T, Stastny P, Zahálka F, et al. Performance and Kinematic Differences in

11. Davids K, Glazier P, Araújo D, et al. Movement systems as dynamical systems: The functional role of variability and its implications for sports medicine. Sports Medicine 2003; 33: 245-260.

12. Bradshaw EJ, Maulder PS, Keogh JWL. Biological movement variability during the sprint start: performance enhancement or hindrance? Sports Biomech 2007; 6: 246-260.

13. Hurrion., \& MacKay J. A Rolling Brief. Golf International, 2012, pp. 107-111. 
14. Karlsen J, Smith G, Nilsson J. The stroke has only a minor influence on direction consistency in golf putting among elite players. J Sports Sci 2008; 26: 243-250.

15. Langdown BL, Bridge M, Li F-X. Movement variability in the golf swing. Sport Biomech 2012; 11: 273-287.

16. Horan SA, Evans K, Kavanagh JJ. Movement variability in the golf swing of male and female skilled golfers. Med Sci Sports Exerc 2011; 43: 1474-1483.

17. Healy A, Moran KA, Dickson J, et al. Analysis of the 5 iron golf swing when hitting for maximum distance. J Sports Sci 2011; 29: 1079-1088.

18. Pelz DT. Dave Pelz's Putting Bible. New York: Random House, 2000.

19. Knight CA. Neuromotor issues in the learning and control of golf skill. Res $Q$ Exerc Sport 2004; 75: 9-15.

20. The R\&A. Rules Of Golf

Definitionshttps://www.randa.org/RulesEquipment/Rules/Rules-Of-GolfDefinitions/\#hole (2018, accessed 16 February 2018).

21. Coleman SGS, Rankin AJ. A three-dimensional examination of the planar nature of the golf swing. J Sports Sci 2005; 23: 227-234.

22. Wheat JS, Vernon T, Milner CE. The measurement of upper body alignment during the golf drive. J Sports Sci 2007; 25: 749-55.

23. Giakas G, Baltzopoulos V, Bartlett RM. Improved extrapolation techniques in recursive digital filtering: A comparison of least squares and prediction. $J$ Biomech 1997; 31: 87-91.

24. Lin WH, Liu YF, Hsieh CCC, et al. Ankle eversion to inversion strength ratio and static balance control in the dominant and non-dominant limbs of young adults. $J$ Sci Med Sport 2009; 12: 42-49.

25. Delphinus EM, Sayers MGL. Putting proficiency: contributions of the pelvis and trunk. Sport Biomech 2012; 11: 212-22.

26. McLaughlin P, Best R. Taxonomy of golf putting: do different golf putting techniques exist? J Sports Sci 2013; 31: 1038-44.

27. Ball K, Best R. Centre of pressure patterns in the golf swing: individual-based analysis. Sport Biomech 2012; 11: 175-189. 


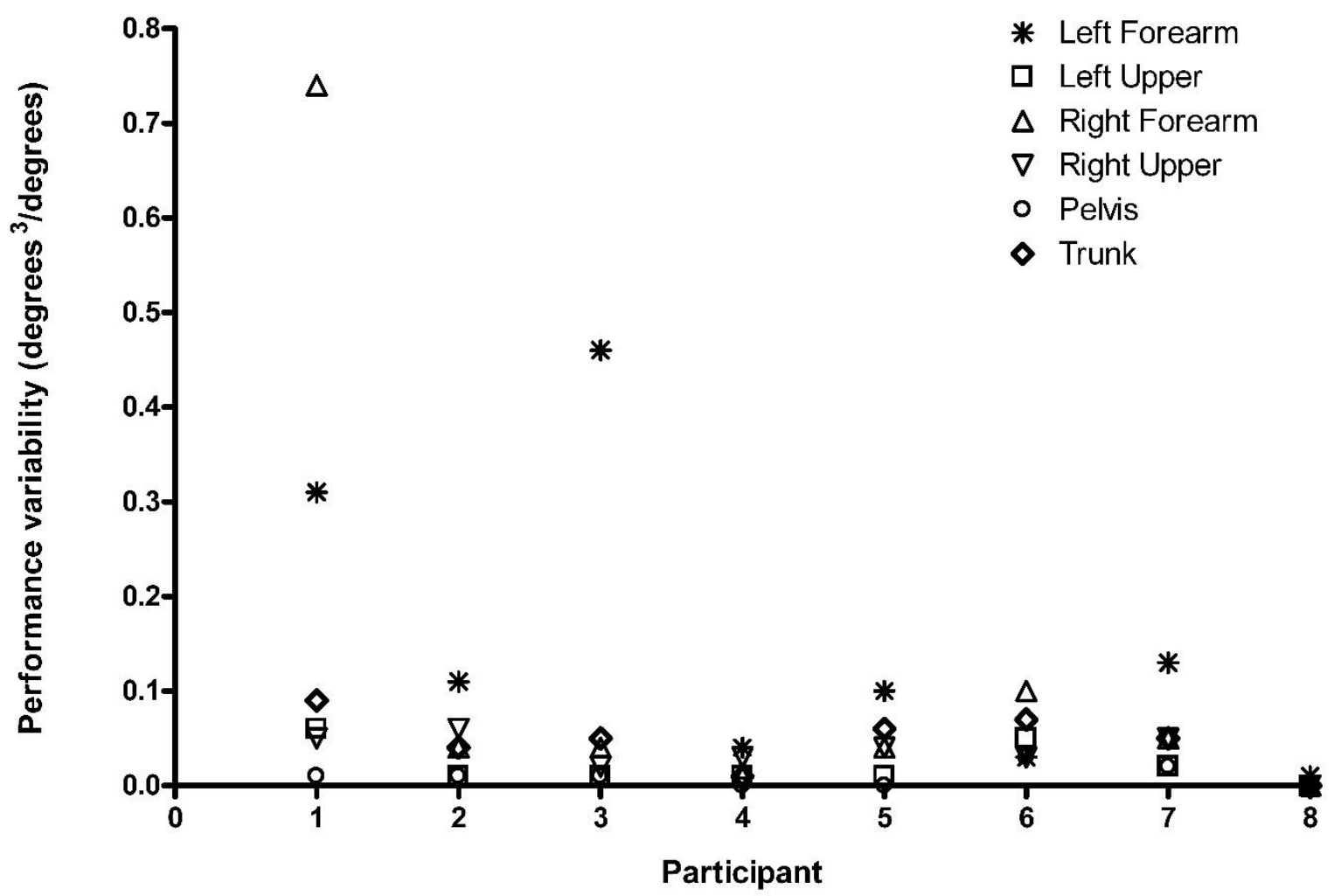

416

417 Figure 1. Scatterplot of performance variability scores for the segment rotations during

418 the putting stroke

419

420

421

422

423

424

425 
427 Table 1. Performance variability scores for the putter $Z$ rotations during the putting stroke 428 and the putter face angle at address.

\begin{tabular}{|c|c|c|c|c|}
\hline Participant & $\begin{array}{c}\text { Playing } \\
\text { Handicap }\end{array}$ & $\begin{array}{l}\text { Performance } \\
\text { variability } \\
\text { (degrees) }\end{array}$ & $\begin{array}{l}\text { Variability of } \\
\text { face angle at } \\
\text { address } \\
\text { (degrees) }\end{array}$ & $\begin{array}{c}\text { Putting } \\
\text { Proficiency } \\
\text { (Success Rate } \\
\%)\end{array}$ \\
\hline 1 & 8 & 0.35 & 0.75 & 73 \\
\hline 2 & 10 & 0.29 & 0.73 & 75 \\
\hline 3 & 5 & 0.18 & 0.67 & 52 \\
\hline 4 & 10 & 0.15 & 0.48 & 71 \\
\hline 5 & 6 & 0.20 & 1.77 & 76 \\
\hline 6 & 0 & 0.26 & 0.54 & 59 \\
\hline 7 & 6 & 0.24 & 0.70 & 67 \\
\hline 8 & 3 & 0.17 & 0.66 & 83 \\
\hline
\end{tabular}


435 Table 2. Correlation coefficients $(r(p))$ between putting proficiency and segment rotation 436 variability.

\begin{tabular}{cccccccccc}
\hline & \multicolumn{8}{c}{ Segment Variability } \\
\cline { 2 - 9 } & Left & Right & Left & Right & & & & \\
& & & Upper & Upper & Pelvis & Trunk & Putter & Face \\
& Forearm & Forearm & Arm & Arm & & & & Angle \\
& & & & & & & & & \\
\hline Putting & -.56 & .02 & -.27 & -.61 & -.44 & -.38 & .03 & .30 \\
Proficiency & $(.15)$ & $(.97)$ & $(.52)$ & $(.11)$ & $(.28)$ & $(.35)$ & $(.94)$ & $(.46)$ \\
\hline
\end{tabular}


438 Table 3. Ball roll kinematic variables for all participants (mean \pm SD).

\begin{tabular}{|c|c|c|c|c|c|c|}
\hline Participant & Velocity $\left(\mathrm{m} \cdot \mathrm{s}^{-1}\right)$ & $\begin{array}{l}\text { Spin (Cut }(+) \text {, } \\
\text { Hook }(-), \text { rpm) }\end{array}$ & $\begin{array}{l}\text { Initial Ball Roll } \\
\qquad(\mathrm{rpm})\end{array}$ & $\begin{array}{c}\text { Forward } \\
\text { Rotation }(\mathrm{cm})\end{array}$ & $\begin{array}{c}\text { Vertical } \\
\text { Launch Angle } \\
\left(^{\circ}\right)\end{array}$ & $\begin{array}{c}\text { Horizontal } \\
\text { Launch Angle }\left(^{\circ}\right)\end{array}$ \\
\hline 1 & $2.28 \pm 0.09$ & $19 \pm 17$ & $65 \pm 14$ & $0.0 \pm 0.1$ & $2.0 \pm 1.1$ & $1.9 \pm 1.7$ \\
\hline 2 & $2.11 \pm 0.09$ & $-20 \pm 11$ & $10 \pm 17$ & $1.9 \pm 2.3$ & $4.3 \pm 0.6$ & $0.2 \pm 0.7$ \\
\hline 3 & $2.08 \pm 0.11$ & $34 \pm 10$ & $38 \pm 12$ & $0.1 \pm 0.2$ & $3.1 \pm 0.6$ & $-1.3 \pm 0.9$ \\
\hline 6 & $2.22 \pm 0.08$ & $-13 \pm 8$ & $-31 \pm 10$ & $9.0 \pm 1.8$ & $5.6 \pm 0.8$ & $1.4 \pm 1.2$ \\
\hline 7 & $2.27 \pm 0.16$ & $-6 \pm 17$ & $16 \pm 11$ & $2.2 \pm 2.8$ & $3.5 \pm 0.7$ & $0.1 \pm 1.1$ \\
\hline 8 & $2.26 \pm 0.07$ & $-4 \pm 11$ & $-16 \pm 11$ & $5.4 \pm 4.5$ & $5.5 \pm 0.9$ & $2.3 \pm 1.2$ \\
\hline
\end{tabular}

439 Key: Cut Spin refers to clockwise rotation and Hook Spin anti-clockwise rotation; a positive Vertical Launch Angle refers the

440 the trajectory of the ball in the $\mathrm{Z}$ axis; a negative Horizontal Launch Angle refers to the trajectory of the ball moving left of 
441 the intended target line and a positive Horizontal Launch Angle refers to the trajectory of the ball moving right of the intended

442 target line.

443 Table 4. Correlation coefficients $(r(p))$ between performance measures variability and segment rotation variability.

\begin{tabular}{|c|c|c|c|c|c|c|c|}
\hline & Left Forearm & $\begin{array}{c}\text { Right } \\
\text { Forearm }\end{array}$ & $\begin{array}{c}\text { Left Upper } \\
\text { Arm }\end{array}$ & $\begin{array}{c}\text { Right Upper } \\
\text { Arm }\end{array}$ & Pelvis & Trunk & $\begin{array}{l}\text { Putter (Z } \\
\text { rotations) }\end{array}$ \\
\hline Velocity & $.03(.95)$ & $.29(.48)$ & $-.31(.45)$ & $-.13(.77)$ & $-.02(.95)$ & $-.16(.71)$ & $-.38(.35)$ \\
\hline Side Spin & $-.45(.26)$ & $.69(.06)$ & $-.17(.70)$ & $-.01(.98)$ & $.10(.82)$ & $.01(.98)$ & $.33(.42)$ \\
\hline Initial Ball Roll & $-.45(.27)$ & $-.04(.93)$ & $-.51(.20)$ & $.01(.99)$ & $-.42(.31)$ & $-.65(.08)$ & $-.16(.70)$ \\
\hline Forward Roll & $-.54(.17)$ & $.00(1.0)$ & $-.48(.23)$ & $-.05(.91)$ & $-.32(.44)$ & $-.68(.06)$ & $-.20(.63)$ \\
\hline $\begin{array}{c}\text { Vertical } \\
\text { Launch Angle }\end{array}$ & $.111(.79)$ & $.23(.58)$ & $.23(.59)$ & $-.48(.22)$ & $.07(.86)$ & $.34(.41)$ & $.10(.82)$ \\
\hline $\begin{array}{l}\text { Horizontal } \\
\text { Launch Angle }\end{array}$ & $.92(<.01)^{*}$ & $-.09(.83)$ & $.11(.79)$ & $.16(.70)$ & $.65(.08)$ & $.42(.30)$ & $-.10(.98)$ \\
\hline
\end{tabular}

(Significant relationship $\left.{ }^{*}, p<.05\right)$.

445 